\title{
CT-guided percutaneous minimally invasive radiofrequency ablation for the relief of cancer related pain from metastatic non-small cell lung cancer patients: a retrospective study
}

\author{
Xiaoyun Zhou ${ }^{1,2 \#}$, Hongsen $\mathrm{Li}^{1 /}$, Qing Qiao ${ }^{3}$, Hongming $\operatorname{Pan}^{1}$, Yong Fang ${ }^{1}$ \\ ${ }^{1}$ Department of Medical Oncology, Sir Run Run Shaw Hospital, Zhejiang University, Hangzhou, China; ${ }^{2}$ Department of Medical Oncology, Sir \\ Run Run Shaw Hospital, Xiasha Campus, Hangzhou, China; ${ }^{3}$ Department of Anesthesiology, Sir Run Run Shaw Hospital, Zhejiang University, \\ Hangzhou, China \\ Contributions: (I) Conception and design: X Zhou, H Li; (II) Administrative support: Q Qiao; (III) Provision of study materials or patients: H Pan; (IV) \\ Collection and assembly of data: Y Fang; (V) Data analysis and interpretation: X Zhou; (VI) Manuscript writing: All authors; (VII) Final approval of \\ manuscript: All authors. \\ "These authors contributed equally to this work. \\ Correspondence to: Hongming Pan, Yong Fang. Department of Medical Oncology, Sir Run Run Shaw Hospital, Zhejiang University, No. 3 East \\ Qingchun Road, Hangzhou 310016, China. Email: panhongming@zju.edu.cn; fangyong@zju.edu.cn.
}

Background: Radiofrequency ablation (RFA) is the current gold standard for palliative care of non-small cell lung cancer (NSCLC). Pain relief for advanced metastases of NSCLC is notoriously difficult. Combined RFA therapy may be more effective than palliating therapy alone in management of painful metastatic disease. The effects of RFA on quality of life, particularly pain, as well as long-term outcome studies are not well studied. To study the effectiveness of percutaneous minimal invasive RFA in pain management of NSCLC patients with metastatic chest wall, vertebral bodies and rib, and periphery lung nodule.

Methods: Forty patients with 59 tumors underwent percutaneous computed tomography (CT) or ultrasound-guided RFA for pain management over a 4-week observation. Forty patients were referred to ablation because of persistent severe pain despite using analgesics, chemotherapy or radiotherapy. The tumors were located in the periphery lung, or metastatic to chest wall, rib or vertebral body. Quantitative pain scale values were quantified on a $0-10$ scale before, 24 hours, 72 hours, and 4 weeks after RFA. On the basis of changes in pain score and pain medication use, pain was reported with a composite measure as complete, partial, or no pain response. The overall survival (OS) rate was also collected and calculated with Kaplan-Meier method.

Results: After 4-week follow-up, complete pain relief (pain scale score $\leq 1$ ) was observed in 12 patients $(30 \%)$ and partial pain relief (pain scale score $\leq 3$ ) in 15 (37.5\%) patients; pain relief did not occur in 13 patients (32.5\%). There was a significant decrease in pain at 24-hour, 72-hour, and 4-week follow-up compared with pain level at baseline $(\mathrm{P}<0.01)$. Opiate use was decreased in $92.5 \%(37 / 40)$ patients, remained unchanged in $7.5 \%(3 / 40)$ at 4 weeks follow-up. There are minor adverse events caused by RFA therapy, including pleural effusion (5/40), post procedural infections (3/40), pneumothorax (2/40) which resolved spontaneously. The OS rates at 6 months in the percutaneous RFA group were $60 \%$, with average OS of 6.5 months in the further follow-up.

Conclusions: Percutaneous RFA resulted in sustained pain relief from in most advanced NSCLC patients with intractable pain and resistant to chemotherapy or radiotherapy. The effect of RFA was satisfactory, and patients can obtain a better life quality with less pain and complications.

Keywords: Non-small cell lung cancer (NSCLC); metastasis, CT-guided percutaneous radiofrequency ablation; cancer related pain; pain relief 
Submitted Nov 08, 2019. Accepted for publication Sep 17, 2020.

doi: 10.21037/apm-19-474

View this article at: http://dx.doi.org/10.21037/apm-19-474

\section{Introduction}

About $30-45 \%$ of patients diagnosed with non-small cell lung cancer (NSCLC) each year in China are failed to be surgical candidates, either due to advanced disease or problems, such as age and comorbidities (1). The " 3 ladder approach" for pain management is effective in the majority $(69-100 \%)$ of patients with cancer pain (2). However, failure does occur, especially in advanced NSCLC patients when conventional palliative treatments such as surgery, radiation therapy (3-5), resistance to tyrosine kinase inhibitor (TKI) (6) and chemotherapy (7) are difficult or contraindicated.

Radiofrequency ablation (RFA) is the treatment to malignant tumors by inserting an electrode into the tumor and causing the thermocoagulation necrosis of the tumor (8). Studies during the past decade (9) and our previous clinical work (10) have showed the emergence of using minimally invasive therapies as the best developed palliative control of cancer pain, with low risk of complications and technically highly successful. It has been documented experimentally and clinically that when RFA was used in combination with other treatments (11), they act synergistically to provide a better management for cancer-related pain (12). With refinements in technology, patient selection and clinical applications, RFA has been used as a potentially viable stand-alone or complementary therapy for both primary (13) and secondary lung malignancies (8) in standard and highrisk populations. Percutaneous RFA is beginning to be used in the management of cancer-related pain, especially in patients with osseous malignancies $(14,15)$. Palliative "debulking" of tumor masses with RFA could results in pain relief significantly (16). However, there are scant data on palliative treatment for pain related to advanced NSCLC with RFA.

In this study, we used RFA combined with other supporting treatments on advanced NSCLC patients suffered from cancer-associated pain, who failed on chemotherapy, radiotherapy or tyrosine-kinase inhibitor (TKI) treatment. The goal of our study was to study the effectiveness of percutaneous RFA in treating cancer-related pain in NSCLC patients.

\section{Methods}

\section{Patients}

This retrospective study was approved by Ethical Committee of Sir Run Run Shaw Hospital (No. 20200617-33) and conducted in accordance with the Declaration of Helsinki (as revised in 2013). The treatment plan and data collection were approved by Ethical Committee of our institution. Participants gave informed consent before taking part. The pathologic diagnosis of NSCLC with core biopsy or surgery specimens was made based on the criteria of World Health Organization (WHO) Classification of Tumours of the Lung, Pleura, Thymus and Heart in 2004. Medical records of all 40 advanced NSCLC patients with pain who underwent percutaneous RFA in this study at our institution between January 01, 2012 and July 01, 2016 were reviewed. Before RFA, each patient was discussed in a multidisciplinary tumor (MDT) board conference before the appropriate approach was decided, including Dept. of Medical Oncology, Radiation Oncology, Radiology, Thoracic surgery, and Intervention. Concurrent treatment was defined as less than 4 weeks post-RFA treatment.

Inclusion criteria includes: (I) individuals over 18 years with advanced NSCLC with pathological confirmation when conventional palliative treatments such as surgery, radiation therapy, tyrosine kinase inhibitor (TKI) and chemotherapy are difficult or contraindicated; (II) any solitary tumor $\leq 7 \mathrm{~cm}$ in diameter and no more than three tumor nodules; (III) moderate or serious pain with the numerical rating scale (NRS) $\geq 4$ with appropriate analgesics; (IV) liver function equal or better than Pugh-Child Class B; and $(\mathrm{V})$ platelet count $>80 \times 10^{9} / \mathrm{L}$ without clinical significant portal hypertension and esophageal varices.

\section{Procedures}

The 59 tumors in 40 patients were identified in the following locations: 21 in the chest wall, 6 in the thoracic vertebral body, 8 in the rib and 24 in the periphery carcinoma nodule. Tumor diameter ranged from 1.46 to $5.00 \mathrm{~cm}$, with a mean of $3.16 \mathrm{~cm}$ (Table 1). RFA was performed when lesions 
Table 1 Forty patients treated with radiofrequency ablation

\begin{tabular}{|c|c|c|c|c|}
\hline Histologic origin of NSCLC & No. of patients & Site of tumor & No. of tumor & Average of tumor size $(\mathrm{cm})$ \\
\hline \multirow{3}{*}{ Adenocarcinoma } & & Vertebral body & 2 & \\
\hline & & $\mathrm{Rib}$ & 6 & \\
\hline & & Periphery tumor nodule & 12 & \\
\hline \multirow{3}{*}{ Squamous cell carcinoma } & & Vertebral body & 3 & \\
\hline & & Rib & 2 & \\
\hline & & Periphery tumor nodule & 10 & \\
\hline Large cell carcinoma & 3 & Chest wall & 2 & $3.06 \pm 1.67$ \\
\hline
\end{tabular}

Table 2 Categorical response to treatment after concurrent RT and ablation

\begin{tabular}{lcccc}
\hline \multirow{2}{*}{ Modality } & No. of patients & \multicolumn{2}{c}{ Pain response at the end of the study period } \\
\cline { 3 - 4 } & 17 & Complete & Partial & None \\
RFA & 13 & 6 & 5 & 4 \\
RFA and chemotherapy & 2 & 4 & 1 & 3 \\
RFA and radiotherapy & 8 & 2 & 3 & 1 \\
RFA and TKI & 40 & 12 & 15 \\
Total & & 0 & 13 \\
\hline
\end{tabular}

RFA, radiofrequency ablation; TKI, tyrosine kinase inhibitor.

were $\leq 5 \mathrm{~cm}$ in diameter and no more than 3 carcinoma nodules with informed consents. All RFA procedures were performed by a dedicated team from the department of medical oncology, using a standard protocol of TYCO RFA device (Valley Lab, Tyco Health Care Group, Boulder, CO, USA) or Rita RFA device (Oncology Products Group Angio Dynamics, Latham, NY, USA) under the guidance of ultrasound (12 patients) or computed tomography scan (Somatom Plus 4; Siemens Medical Solutions, Forchheim, Germany) (28 patients). Patients received the procedure under general anesthesia. RFA electrode was guided toward tumors and each tumor was treated with a single electrode placement and just one ablation. To ensure ablation of most of the tumor, multiple ablation zones were used with a mean of 8 overlapping ablation zones per treated level. Chest CT scan was performed at the end of the RFA to rule any active hemorrhage or pneumothorax. After RFA, other combined treatments were used in 23 patients, including chemotherapy in 13 patients, radiotherapy in 2 patients, TKI treatment in 8 patients because of pain symptom relief with improved quality of life (Table 2).

\section{Post-treatment follow-up, pain score and CT scans}

Follow-up visits after ablations were scheduled with oncologists. Medical records were reviewed to assess the level of pain on a $0-10$ scale before ablation. Mean pain scores were calculated for 4 group, including Pre-RFA procedure, 24 hours, 72 hours, and 4 weeks after the RFA procedure. Intravenous opiates for pain were used as needed in the first 2-3 hours after RFA. But patients were restarted on their baseline analgesic regimen when oral intake was resumed.

Analgesic histories were recorded. Overall degree of pain relief based on quantitative pain scale results were reported 
in this study with a composite measurement that combined the change in pain medication and pain score, both of which were assessed at 4 weeks follow-up. Subsequent pain scores, changes in general activity and analgesic opiates medication use were obtained via telephone interview or through patient visit 4 weeks after the procedure. Analgesic medication use was defined as more, same, or less absolute $\mathrm{mg} /$ day. The pain response after 4 weeks follow-up were denoted as follows: (I) complete pain response, with total relief of pain without further use of analgesic medications; (II) partial pain response, defined with use of the same analgesic medication regimen or an palliative pain score to allow a decrease in the use of pain medication; and (III) no pain response, defined with recurrence of pain that reached pre procedural levels, or post procedural pain that necessitated an increase in the use of pain medication beyond pre procedural levels.

All adverse events, including the treatment related pain, were recorded and compared. Forty patients were evaluated via contrast-enhanced CT (SOMATOMAR-T CT scanner) at the 4 weeks post RFA with contrast material (100 mL intravenous iopromide, Ultravist 300; Berlex Laboratories, Madison, NJ, USA). Necrosis was defined as the unenhanced area on the postprocedural CT images. Complete ablation was defined as the absence of any peripheral enhancement in the contrast-enhanced phase 4 weeks after the RFA treatment. Local recurrence was defined as recurrences contiguous to or within ablated areas. Distant intra-lung recurrence was defined as a new tumor that appeared in the lung away from the ablated area.

\section{Statistical analysis}

Results were given as mean \pm standard deviation. Quantitative pain scale scores (obtained before the procedure, 24 hours, 72 hours and 4 weeks post-RFA) were compared by using Friedman repeated-measures one-way analysis of variance $(\mathrm{P}<0.050)$. The $t$-test was used for continuous variables in a parametric fashion. The Kaplan-Meier method was used to estimate the cumulative incidences of events. All statistical evaluations were performed using the SPSS 21.0 software package (SPSS Inc., Chicago, IL, USA). All statistical tests were two-sided, and a significant difference was considered when $\mathrm{P}<0.05$.

\section{Results}

Patients and treatments: the study included 28 men and
12 women, with a median age of $55.6 \pm 16.8$ years (range, 41-73). All patients were Chinese. As Table 1 showed, the most common types of primary tumor of NSCLC were adenocarcinoma $(52.5 \%, 21 / 40)$ and squamous cell carcinoma $(40 \%, 16 / 40)$, with the average of tumor size $3.23 \pm 1.84$ and $2.74 \pm 1.56 \mathrm{~cm}$, respectively. Large cell carcinoma $(7.5 \%, 3 / 40)$ is the least common type with the average of tumor size $3.06 \pm 1.67 \mathrm{~cm}$.

As Table 2 showed, two patients received radiotherapy treatment post-RFA. One patient accepted stereotactic body radiation therapy (SBRT) for vertebral body with pathological fractures, and the other accepted SBRT for mediastinal tumor with hemoptysis. And 13 chemotherapy and 8 TKI targeted treatments were performed after RFA. One lesion $(2.5 \% ; 1 / 40)$ was re-treated with RFA for the recurrent pain and imaging evidence of tumor.

\section{RFA significantly decreased the pain score and opiates usage}

As shown in Figure $1 A$ and Table 3, the pre-RFA mean worst pain score $(7.60 \pm 2.22)$ significantly decreased at both 24 hours $(3.20 \pm 1.75, \mathrm{P}<0.001), 72$ hours $(2.10 \pm 1.23, \mathrm{P}<0.001)$ and 4 -week $(3.50 \pm 1.68, \mathrm{P}<0.001)$ following ablation treatments, compared to the mean pre-procedural VAS score. Patient's opiate usage was decreased by $92.5 \%$ (37/40), during the following-up 4 weeks (Figure 1A).

Complete pain response (pain scale score $\leq 1$ ) and partial pain response (pain scale score $\leq 3$ ) were $30 \%(12 / 40)$ and $37.5 \%(15 / 40)$, respectively, after 4 weeks (Table 4$)$. No significant relief was also noticed at 24 hours $2.5 \%(1 / 40)$, 72 hours $2.5 \%$ (1/40) and 4 weeks $7.5 \%(3 / 40)$.

\section{Complications of RFA were tolerable}

There are no major complication, such as bleeding, permanent neural thermal injury, occurred as followed by lung percutaneous RFA. There were minor complications caused by RFA, including 5 pleural effusion, 3 post procedural infection, 2 pneumothorax which resolved spontaneously.

\section{Prognosis of patients receiving $R F A$}

All of the patients had post-procedure imaging evaluation at least 1 month, consisting of CT, magnetic resonance imaging (MRI), and/or positron emission tomography (PET)/CT. As Figure $1 B$ showed, the overall survival (OS) 

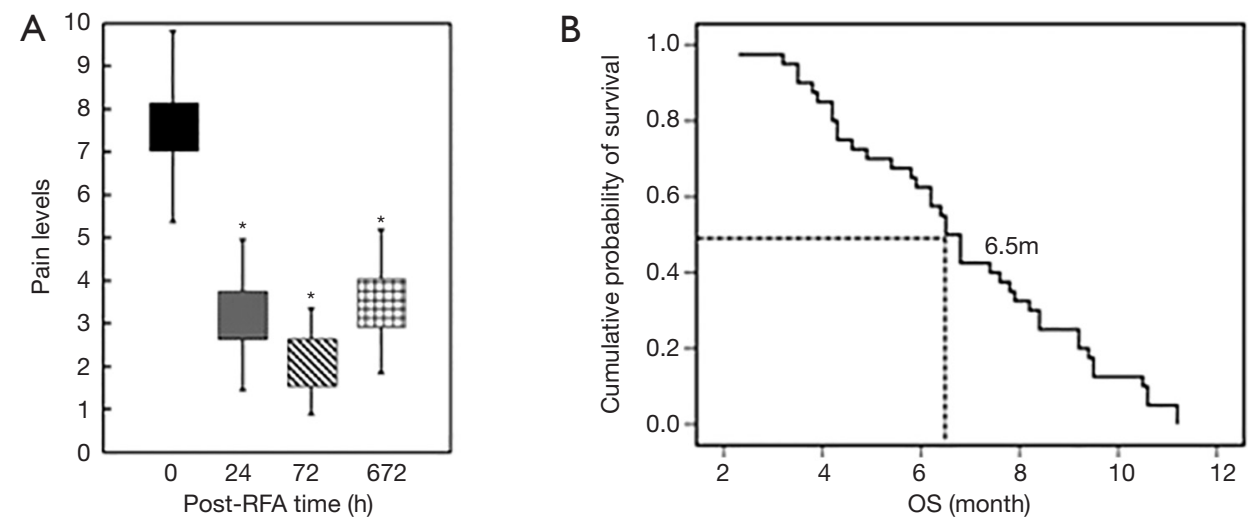

Figure 1 The average of peak pain score at 0, 24, 72 and $672 \mathrm{~h}$ after RFA treatment. (A) Pain scale response following RFA treatment in advanced NSCLC. (B) The average of overall survival rates. * ${ }^{*}$ pompared with the average of peak pain score at $0 \mathrm{~h}$ after RFA treatment.

Table 3 VAS score and pain medication usage

\begin{tabular}{lc}
\hline Parameter & Value \\
\hline Pre-RFA VAS score & $7.60 \pm 2.22$ \\
24 h post-RFA VAS score & $3.20 \pm 1.75^{\star}$ \\
72 h post-RFA VAS score & $2.10 \pm 1.23^{\star}$ \\
4 w post-RFA VAS score & $3.50 \pm 1.68^{\star}$ \\
Decrease in pain medication use (\%) & $92.5(37$ of 40$)$ \\
Unchanged pain medication use (\%) & $7.5(3$ of 40$)$
\end{tabular}

*, $\mathrm{P}<0.01$ based on the matched two-tailed Student's $t$-test. RFA, radiofrequency ablation.

Table 4 VAS scores and pain medication usage

\begin{tabular}{|c|c|c|c|c|c|}
\hline $\begin{array}{l}\text { Degree of relief based on } \\
\text { quantitative pain scale }\end{array}$ & $\begin{array}{l}\text { Pain relief within } \\
\qquad 24 \mathrm{~h}(\mathrm{n})\end{array}$ & $\begin{array}{l}\text { Pain relief within } \\
72 \mathrm{~h}(\mathrm{n})\end{array}$ & \multicolumn{3}{|c|}{ Pain relief at the end of study period } \\
\hline$\geq 50 \%$ & 26 & 35 & 9 & 12 & 6 \\
\hline$<50 \%$ & 13 & 4 & 3 & 3 & 4 \\
\hline No relief of worse pain & 1 & 1 & 0 & 0 & 3 \\
\hline
\end{tabular}

rates at 6 months in the percutaneous RFA group were $60 \%$, and the average OS in these 40 advanced NSCLC patients was 6.5 months, despite of systemic progression disease (PD). There was imaging evidence of tumor progression at the ablation margins in 4 patients at 6 months. One patient with less than 3 months had progressive vertebral compression, and 3 patients had tumor progression at 8 weeks after concurrent therapy. One female patient received 2 RFA treatments for 2 periphery carcinoma nodule RFA procedures (Figure 2). This patient's preprocedural pain score $(8 / 10)$ decreased to 0 at 4 weeks follow-up after the 2 nd RFA treatment. The patient had complete response to RFA in the pain control. One male patient received 3 times of RFA for the huge left lung mass located in the upper-lobe, infiltrating mediastinal pleura and chest wall (Figure 3). The patient had partial response 

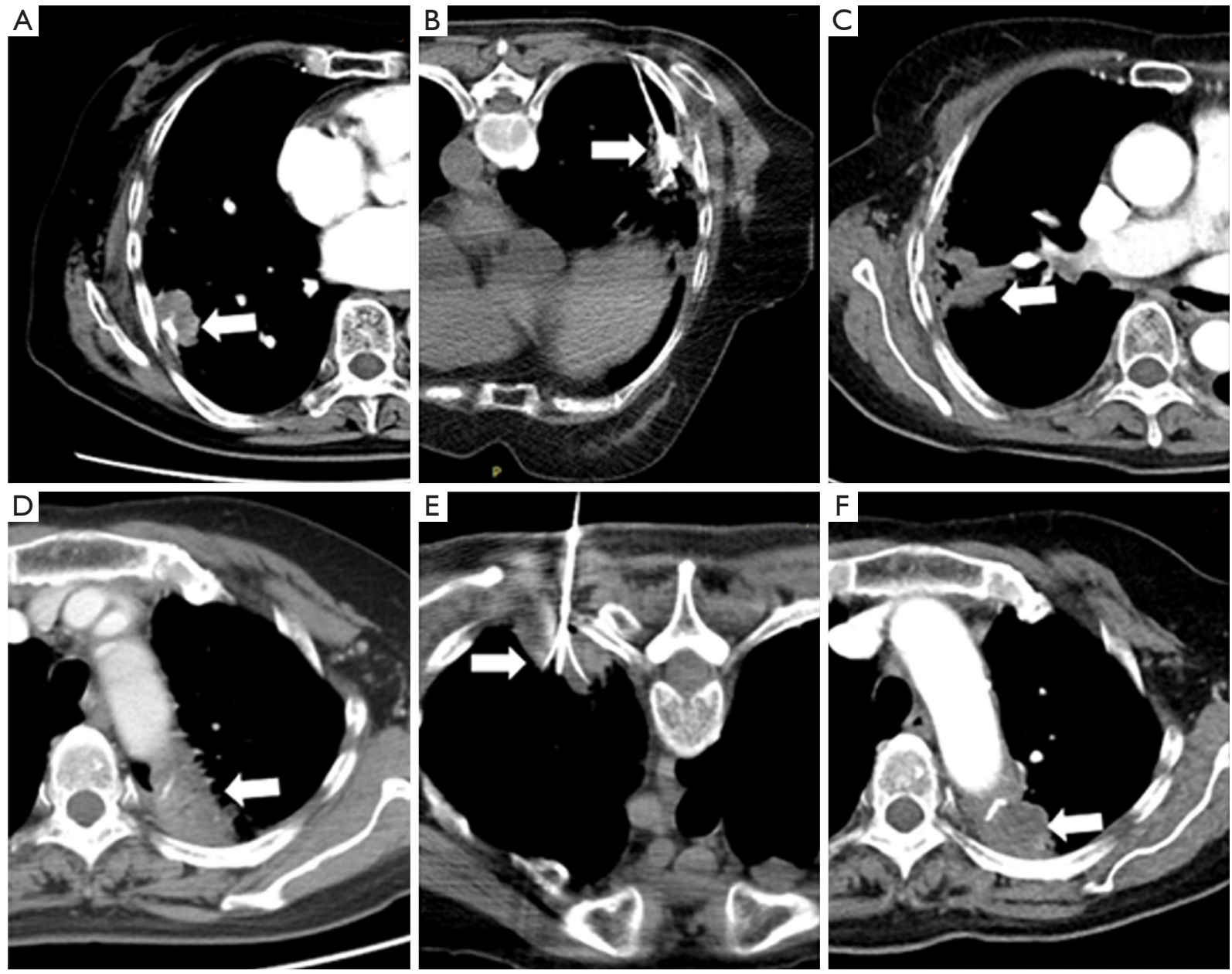

Figure 2 A 59-year-old female with metastatic non-small cell lung adenocarcinoma in the left and right periphery tumor nodule resistant to chemotherapy and radiotherapy, and with serious pathologic pain. (A) One month prior to planned RFA show a pathologic pain in the right periphery tumor nodule. (B) RFA treatment the right-side mass located in the chest wall under CT guidance. (C) Four weeks after RFA showing complete ablation as the absence of peripheral enhancement in the contrast-enhanced phase, and complete response of right chest back pain relief. (D) Two months prior to planned RFA show a pathologic pain in the left periphery tumor nodule. (E) After 1 month of right-side periphery tumor nodule of RFA, RFA treatment of the left side mass located on the chest wall under CT guidance. (F) Four weeks after 2nd RFA showing complete ablation as the absence of peripheral enhancement in the contrast-enhanced phase, and complete response of left chest back pain relief. The patient's pre-procedural pain score [8/10] decreased to 0 at 4 weeks follow-up post 2 nd RFA of left nodule. The patient had complete response to RFA in the pain control after follow-up.

to RFA, and pre-procedural pain score (7/10) decreased to 2 after 4 weeks.

\section{Discussion}

Our study suggested that percutaneous RFA was emerging as a choice of treatment to advanced lung cancer patients who have pain caused by NSCLC. We observed that percutaneous RFA could significantly increase the OS time and decrease the opioid dose after the procedure compared to baseline level pre-RFA $(\mathrm{P}<0.01)$.

Recently, Locklin et al. performed RFA for pain relief in 14 patients with 15 soft-tissue tumors (17). It was found that the feasibility of using RFA for pain relief in 12 patients with painful bone metastasis (18). In these reports, the locations of tumor included the chest wall, breast, and 

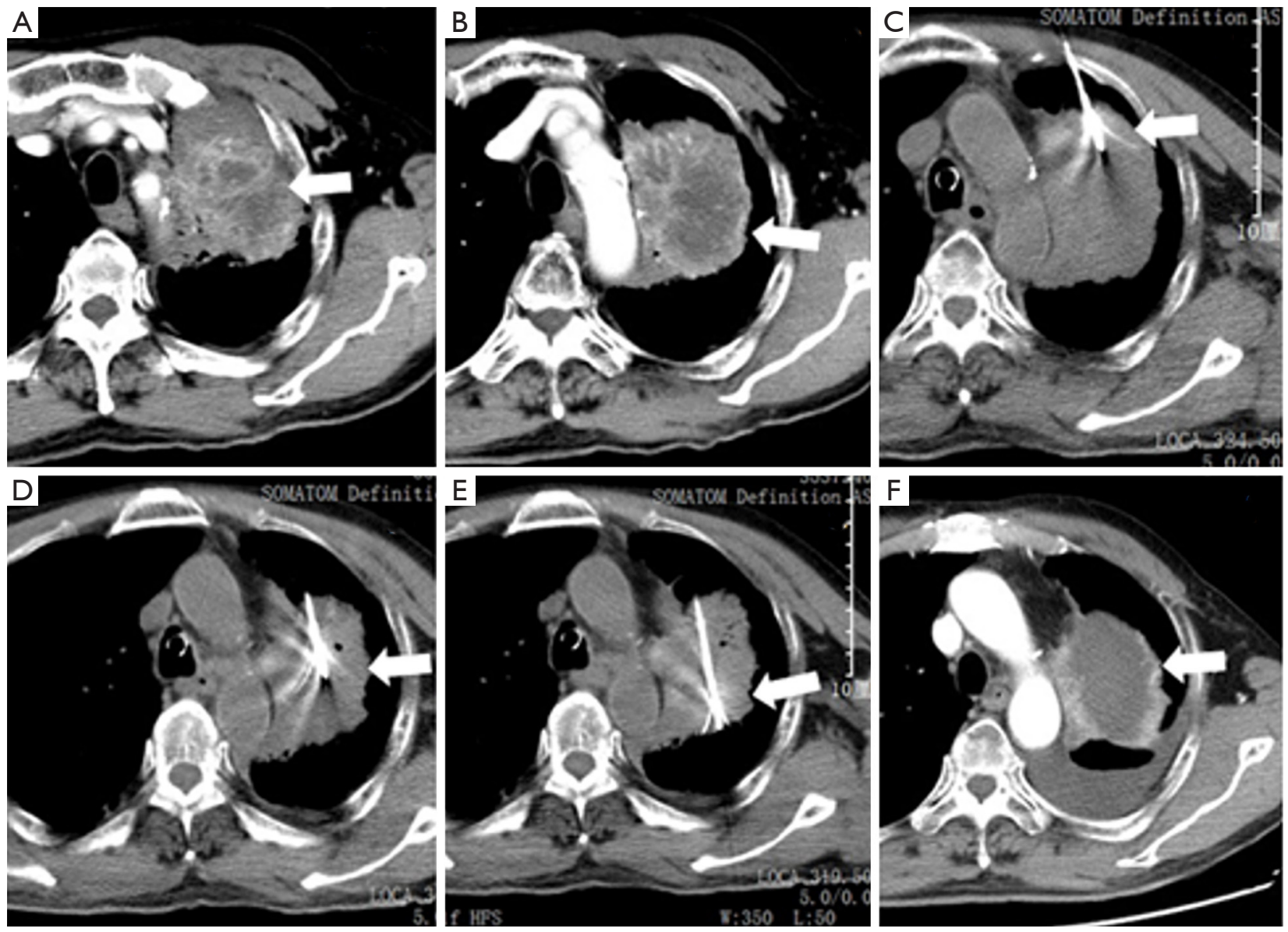

Figure 3 A 61-year-old man with metastatic squamous cell carcinoma resistant to chemotherapy and radiotherapy, suffered with serious pathologic pain. (A,B) of chest CT contrast images scan 2 days prior to RFA showed a huge mass located in the left upper-lobe, rib and mediastinal pleura and chest wall. (C-E) showed 3 times RFA at different location in the same direction one time. (F) Four weeks after RFA show partial ablation as the peripheral enhancement in the contrast-enhanced phase (arrows). The patient's pre-procedural pain score (7/10) decreased to 3 at 4 weeks follow-up post RFA. The patient had partial response to RFA in the pain control after follow-up.

superficial soft tissues, but no information was available on the change in analgesic regimen after ablation therapy. Our results from 40 patients confirms the short-term success of RFA for pain control. It significantly decreased the pain score level for the tumor locating in the chest wall, rib, vertebral body and periphery lung.

Metastases to visceral organs such as the lung and liver are thought to cause pain through capsular distention (19). The mechanism of pain relief after RFA is still not well understood (20). In this study, we focused predominantly on tumor located in chest wall, vertebral body, rib and periphery lung in advanced NSCLC patients including adenocarcinoma, squamous cell carcinoma and large cell carcinoma. However, the tumor size might be expected to be related with pain levels at the first 48 hours after RFA. There is also a significant $(67.5 \%)$ decrease in the pain levels after 4 weeks, including 30\% (12/40 cases) complete pain relief and $37.5 \%$ (15/40 cases) partial relief. The majority of our patients (26/40 cases) experienced significantly relief $(\geq 50 \%)$ based on quantitative pain scale in the first 24 hours, and 35 of 40 (87.5\%) patients experienced a $\geq 50 \%$ decrease within 72 hours. These clinical data suggested that peritumoral denervation (21) or thermal injury to the nerves may be a possible mechanism of pain relief (22), rather than capsular distention (19). Other possible mechanisms including intratumoral pressure and cytoxin release reducing, and decreased pressure on adjacent anatomy (23). In some cases, just creating a small thermal injury on the 
tumor can relieve pain, probably through these speculative mechanisms. The low incidence of procedure-related pain and major complication is consistent with earlier reports on RFA of hepatic tumors in our previous report (10).

In this study, the time course and degree of pain relief allowed us to prove that the palliative RFA procedure had been successful. A significant decrease in quantitative pain scale and pain medication use within the first 72 hours indicated markedly improvement of life quality. Within the first 24 hours, at least $65 \%$ patients' pain score $(3.20 \pm 1.75)$ decreased more than $50 \%$ compared with the preprocedural pain level $(7.60 \pm 2.22)$, and 12 of 40 patients $(30 \%)$ were completely response after 4 weeks. As a result of pain relief, 23 of 40 patients tried to accept other treatments, such as chemotherapy, radiotherapy and TKI. Tumor RFA technologies could generate large size of tumor debris in situ, releasing multiple bio-molecules like tumor antigens and damage-associated molecular patterns (23). RFAinduced tumor-cell necrosis generates an immunogenic source of tumor antigens known to induce antitumor immune responses (24). Therapies combining ablation with immune stimulation are therefore expected to be key to boost RFA-induced immune effects and to achieve systemic, long-lasting, antitumor immunity, which may be helpful in the prevention of the recurrence and improves progressionfree survival for cancer patients $(25,26)$. In our study, the OS rates at 6 months was $60 \%$, with 6.5 months of average OS in the 40 advanced terminal stage of NSCLC patients.

The major limitations of this study included the lack of control subjects, the small sample size, the short time of follow-up. Given the retrospective nature of this study, it was difficult to establish a uniform definition of RFA treatment related analgesic failure.

In summary, our study indicated image-guided RFA was a minimally invasive, safe and effective method and could be promising for pain relief in advanced NSCLC patients who suffered from persistent tumor-related pain. However, it still needs other prospective studies involving matched patient groups with quality-of-life outcomes to convince the treatment effects, and to determine the role of ablation procedures for patients who have cancer-related pain in the future.

\section{Acknowledgments}

Funding: This study was supported by Project of Zhejiang Provincial Natural Science Foundation of China (Grant Number LY13H160013, LQ16H160003), Health
Commission of Zhejiang Province (Grant Number 2016KYA115), Zhejiang Medical and Health Science and Technology Project (Grant Number 2016ZDB007, 2017197380, 2017ZD021), Project of Zhejiang Health and Family Planning Commission (Grant Number 2016151029).

\section{Footnote}

Data Sharing Statement: Available at http://dx.doi. org/10.21037/apm-19-474

Conflicts of Interest: All authors have completed the ICMJE uniform disclosure form (available at http://dx.doi. org/10.21037/apm-19-474). The authors have no conflicts of interest to declare.

Ethical Statement: The authors are accountable for all aspects of the work in ensuring that questions related to the accuracy or integrity of any part of the work are appropriately investigated and resolved. This retrospective study was approved by Ethical Committee of Sir Run Run Shaw Hospital (No. 20200617-33) and conducted in accordance with the Declaration of Helsinki (as revised in 2013). Participants gave informed consent before taking part.

Open Access Statement: This is an Open Access article distributed in accordance with the Creative Commons Attribution-NonCommercial-NoDerivs 4.0 International License (CC BY-NC-ND 4.0), which permits the noncommercial replication and distribution of the article with the strict proviso that no changes or edits are made and the original work is properly cited (including links to both the formal publication through the relevant DOI and the license). See: https://creativecommons.org/licenses/by-nc-nd/4.0/.

\section{References}

1. Choe YH, Kim SR, Lee KS, et al. The use of PTC and RFA as treatment alternatives with low procedural morbidity in non-small cell lung cancer. Eur J Cancer 2009;45:1773-9.

2. Carlson CL. Effectiveness of the World Health Organization cancer pain relief guidelines: an integrative review. J Pain Res 2016;9:515-34.

3. Mulvenna P, Nankivell M, Barton R, et al. Dexamethasone and supportive care with or without whole brain radiotherapy in treating patients with non- 
small cell lung cancer with brain metastases unsuitable for resection or stereotactic radiotherapy (QUARTZ): results from a phase 3 , non-inferiority, randomised trial. Lancet 2016;388:2004-14.

4. Hayashi K, Yamamoto N, Karube M, et al. Feasibility of carbon-ion radiotherapy for re-irradiation of locoregionally recurrent, metastatic, or secondary lung tumors. Cancer Sci 2018;109:1562-9.

5. Ma JT, Zheng JH, Han CB, et al. Meta-analysis comparing higher and lower dose radiotherapy for palliation in locally advanced lung cancer. Cancer Sci 2014;105:1015-22.

6. Qi H, Jiang S, Yu D, et al. Continuing EGFR-TKI treatment in combination with super-selective arterial infusion chemotherapy beyond disease progression for patients with advanced EGFR-mutant non-small cell lung cancer. Med Oncol 2015;32:256.

7. Kuskowski M, Gupta K, Gupta P. Association of opioid requirement and cancer pain with survival in advanced non-small cell lung cancer. J Clin Oncol 2014;32:183.

8. Akhan O, Guler E, Akinci D, et al. Radiofrequency ablation for lung tumors: outcomes, effects on survival, and prognostic factors. Diagn Interv Radiol 2016;22:65-71.

9. Zhang B, Moser MA, Zhang EM, et al. A review of radiofrequency ablation: Large target tissue necrosis and mathematical modelling. Phys Med 2016;32:961-71.

10. Fang Y, Chen W, Liang X, et al. Comparison of long-term effectiveness and complications of radiofrequency ablation with hepatectomy for small hepatocellular carcinoma. J Gastroenterol Hepatol 2014;29:193-200.

11. Greenwood TJ, Wallace A, Friedman MV, et al. Combined Ablation and Radiation Therapy of Spinal Metastases: A Novel Multimodality Treatment Approach. Pain Physician 2015;18:573-81.

12. Cascella M, Muzio MR, Viscardi D, et al. Features and Role of Minimally Invasive Palliative Procedures for Pain Management in Malignant Pelvic Diseases: A Review. Am J Hosp Palliat Care 2017;34:524-31.

13. de Baere T, Tselikas L, Catena V, et al. Percutaneous thermal ablation of primary lung cancer. Diagn Interv Imaging 2016;97:1019-24.

14. Rana H, Matchett G. Using pulsed radiofrequency ablation to treat pain associated with a tumor involving the brachial plexus. Pain Physician 2013;16:E311-4.

15. Stone J, Matchett G. Combined ultrasound and fluoroscopic guidance for radiofrequency ablation of the obturator nerve for intractable cancer-associated hip pain. Pain Physician 2014;17:E83-7.

16. Sahay A, Sahay N, Kapoor A, et al. Percutaneous Image- guided Radiofrequency Ablation of Tumors in Inoperable Patients - Immediate Complications and Overall Safety. Indian J Palliat Care 2016;22:67-73.

17. Locklin JK, Mannes A, Berger A, et al. Palliation of soft tissue cancer pain with radiofrequency ablation. J Support Oncol 2004;2:439-45.

18. Bang JY, Sutton B, Hawes RH, et al. EUS-guided celiac ganglion radiofrequency ablation versus celiac plexus neurolysis for palliation of pain in pancreatic cancer: a randomized controlled trial (with videos). Gastrointest Endosc 2019;89:58-66.e3.

19. Vad VB, Sakalkale D, Warren RF. The role of capsular distention in adhesive capsulitis. Arch Phys Med Rehabil 2003;84:1290-2.

20. Nair RT, vanSonnenberg E, Shankar S, et al. Visceral and soft-tissue tumors: radiofrequency and alcohol ablation for pain relief--initial experience. Radiology 2008;248:1067-76.

21. Ramasubba C, Cohen SP. Cooled sacroiliac radiofrequency denervation for the treatment of pain secondary to tumor infiltration: a case-based focused literature review. Pain Physician 2013;16:1-8.

22. Engel AJ. Utility of intercostal nerve conventional thermal radiofrequency ablations in the injured worker after blunt trauma. Pain Physician 2012;15:E711-8.

23. Xie E, Garzon-Muvdi T, Bender M, et al. Association Between Radiofrequency Rhizotomy Parameters and Duration of Pain Relief in Trigeminal Neuralgia Patients with Recurrent Pain. World Neurosurg 2019;129:e128-33.

24. Gameiro SR, Higgins JP, Dreher MR, et al. Combination therapy with local radiofrequency ablation and systemic vaccine enhances antitumor immunity and mediates local and distal tumor regression. PLoS One 2013;8:e70417.

25. Shi L, Chen L, Wu C, et al. PD-1 Blockade Boosts Radiofrequency Ablation-Elicited Adaptive Immune Responses against Tumor. Clin Cancer Res 2016;22:1173-84.

26. Chiu HY, Leu JD, Chang CY, et al. Combination of Radiofrequency Ablation and Glycated Chitosan as Treatment on a Syngeneic Breast Tumor Model. Anticancer Res 2017;37:2965-74.

Cite this article as: Zhou X, Li H, Qiao Q, Pan H, Fang Y. CT-guided percutaneous minimally invasive radiofrequency ablation for the relief of cancer related pain from metastatic non-small cell lung cancer patients: a retrospective study. Ann Palliat Med 2021;10(2):1494-1502. doi: 10.21037/apm-19-474 